\title{
Renal Solitary Fibrous Tumor with Thrombus Extension into the Inferior Vena Cava and Right Atrium
}

\author{
Farouk Almohammad ${ }^{1}$ and Mohamad Mohamad Bakour ${ }^{2}$ \\ ${ }^{1}$ Almajd Specialized Hospital \\ ${ }^{2}$ Almajd specialized Hospital
}

September 22, 2020

\begin{abstract}
Background A solitary fibrous tumor (SFT) of the kidney with thrombus extended into the inferior vena cava (IVC) and right atrium (RA) is very rare. Methods and conclusion We reported here an extremely case of right renal SFT extended to the IVC and RA, treated late with an urgent RA mass removal and IVC thrombectomy.
\end{abstract}

\section{CASE REPORT}

A 35-year-old female who underwent a right nephrectomy for a large right kidney mass month ago now presented with increasing dyspnea and low extremities edema. Computed tomography/angiography showed a large right kidney soft tissue mass, with an intravascular mass filling the inferior vena cava (IVC) above renal veins level up to the right atrium (RA) (Fig. 1). At the time of surgery through median sternotomy and on cardiopulmonary bypass, the right atrium was opened and a free large floating mass was found to protrude from the orifice of the IVC without direct invasion into the myocardium (Fig. 2A). The IVC was explored under a period of deep hypothermic circulatory arrest (DHCA) and residual tumor was excised with intracaval camera assistant (Fig. 2B). Pathologic and immunologic studies confirmed the diagnosis of a malignant form of solitary fibrous tumor (SFT) of the right kidney (staining for CD99, CD34) with thrombus extension into the IVC and RA. The patient was asymptomatic and doing clinically well on follow up to 5 months after surgery and imaging studies revealed $4 \mathrm{~cm}$ right pulmonary node suggesting metastatic progression with no abdominal or intravascular recurrence. Based on newly available data, the patient started a chemotherapy protocol which he is currently receiving with good tolerance.

\section{DISCUSSION}

Renal tumors involve the inferior vena cava (IVC) in almost $5 \%$ to $10 \%$, their thrombus extension to the right atrium occurs at a percentage of $1 \%$, and renal cell carcinoma is the most common of all it ${ }^{1}$. Extrapleuritic solitary fibrous tumors (SFTs) are extremely rare, with about 64 cases of SFTs of the kidney and one case of its extensive vascular thrombus extending to the right atrium (RA) available in the English literature. The first case of renal SFT with thrombus extending to the IVC and RA was treated with a combined abdominalthoracic surgery ${ }^{2}$. Immunohistochemical testing usually helps making the diagnosis of renal SFTs. A $90 \%$ of SFTs are benign with some tumuor characteristics such as necrosis, hemorrhage, size, and vascular thrombus suggesting of malignancy ${ }^{3}$. Systemic chemotherapy, immunotherapy, and radiotherapy may have a role in the treatment of malignant or metastatic disease, but there are currently no standard protocols or regimen ${ }^{4}$.

We reported a case of SFT of the right kidney with thrombus extended into the IVC and RA. The patient was referred to us late after right nephrectomy because of critical IVC and tricuspid valve obstruction symptoms. Urgent RA mass removal and upper IVC thromboctomy performed by DHCA and camera assistant. Early clinical improvement was noted postoperatively after decompressing the right heart. Our case is unique in 
the extent of the associated vascular thrombus, required urgent cardiac surgery and the surgery required for resection is a second in the literature for this type of tumour.

\section{CONFLICT OF INTERESTS}

The authors declare that there are no conflict of interests. Almajd Specialized Hospital approved the case for publication. Informed consent was taken orally from the patient and study participants for publication.

\section{References}

1. Dedeilias, et al. Renal tumor with cavoatrial extension. Card Surg 2009;24:617-623.

2. F. Couture, et al. Malignant solitary fibrous tumour of the kidney with an extensive thrombus: A case report and review of the literature. Urology Case Reports 26 (2019) 100974.

3. Khater N., Khauli R., Shahait M., Degheili J., Khalifeh I., Aoun J. Solitary fibrous tumors of the kidneys: presentation, evaluation, and treatment. Urol Int. 2013;91(4):373-383.

4. Kuroda N., Ohe C., Sakaida N. Solitary fibrous tumor of the kidney with focus on clinical and pathobiological aspects. Int J Clin Exp Pathol. 2014;7(6):2737-2742.

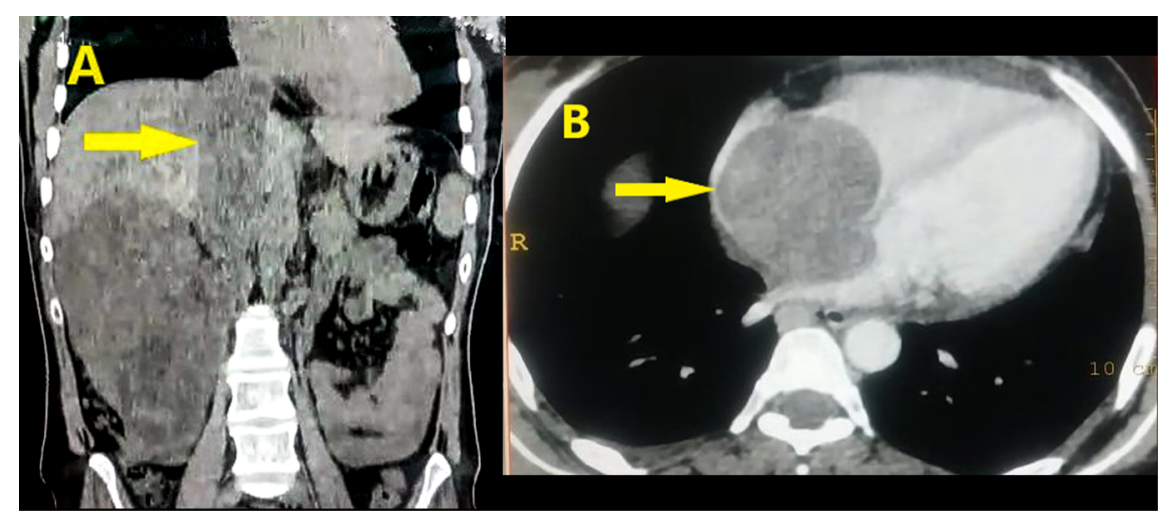




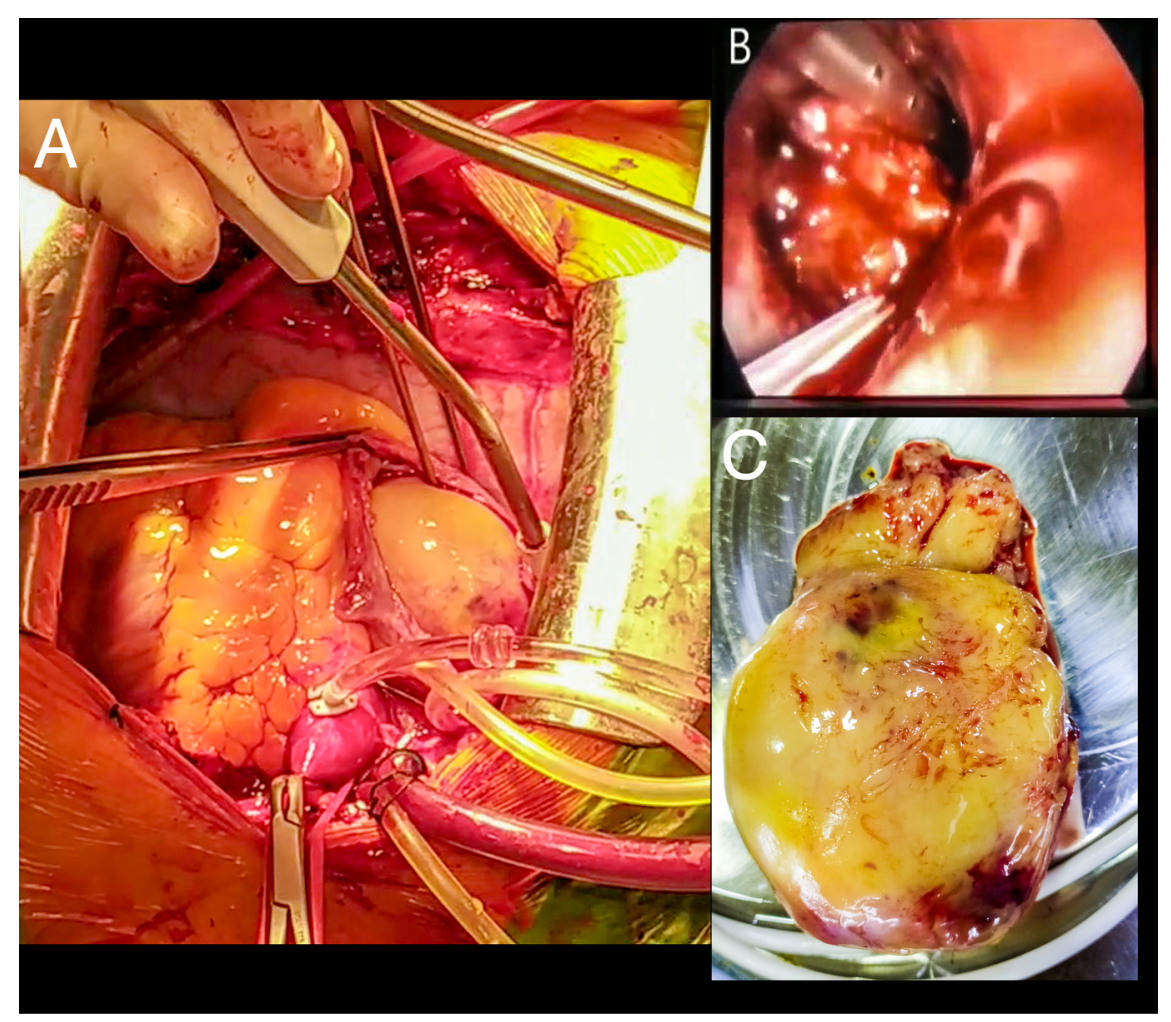

